\title{
Video Article \\ Imaging Cell Interaction in Tracheal Mucosa During Influenza Virus Infection Using Two-photon Intravital Microscopy
}

\author{
Miguel Palomino-Segura* ${ }^{1,2}$, Tommaso Virgilio* ${ }^{1,2}$, Diego Morone ${ }^{1}$, Diego U. Pizzagalli ${ }^{1,3}$, Santiago F. Gonzalez \\ ${ }^{1}$ Faculty of Biomedical Sciences, Institute for Research in Biomedicine, Università della Svizzera italiana (USI) \\ ${ }^{2}$ Graduate School of Cellular and Molecular Sciences, Faculty of Medicine, University of Bern \\ ${ }^{3}$ Institute of Computational Science, Università della Svizzera italiana (USI) \\ * These authors contributed equally
}

Correspondence to: Santiago F. Gonzalez at santiago.gonzalez@irb.usi.ch

URL: https://www.jove.com/video/58355

DOI: doi: $10.3791 / 58355$

Keywords: Immunology and Infection, Issue 138, Two-Photon Intravital Microscopy, Trachea, Influenza virus, Neutrophil, Dendritic Cell, Cell-to-Cell interactions

Date Published: 8/17/2018

Citation: Palomino-Segura, M., Virgilio, T., Morone, D., Pizzagalli, D.U., Gonzalez, S.F. Imaging Cell Interaction in Tracheal Mucosa During Influenza Virus Infection Using Two-photon Intravital Microscopy. J. Vis. Exp. (138), e58355, doi:10.3791/58355 (2018).

\section{Abstract}

The analysis of cell-cell or cell-pathogen interaction in vivo is an important tool to understand the dynamics of the immune response to infection. Two-photon intravital microscopy (2P-IVM) allows the observation of cell interactions in deep tissue in living animals, while minimizing the photobleaching generated during image acquisition. To date, different models for 2P-IVM of lymphoid and non-lymphoid organs have been described. However, imaging of respiratory organs remains a challenge due to the movement associated with the breathing cycle of the animal.

Here, we describe a protocol to visualize in vivo immune cell interactions in the trachea of mice infected with influenza virus using 2P-IVM. To this purpose, we developed a custom imaging platform, which included the surgical exposure and intubation of the trachea, followed by the acquisition of dynamic images of neutrophils and dendritic cells (DC) in the mucosal epithelium. Additionally, we detailed the steps needed to perform influenza intranasal infection and flow cytometric analysis of immune cells in the trachea. Finally, we analyzed neutrophil and DC motility as well as their interactions during the course of a movie. This protocol allows for the generation of stable and bright 4D images necessary for the assessment of cell-cell interactions in the trachea.

\section{Video Link}

The video component of this article can be found at https://www.jove.com/video/58355/

\section{Introduction}

Two-photon intravital microscopy (2P-IVM) is an effective technique for real time imaging of cell-to-cell interactions as they occur in their natural environment ${ }^{1}$. One of the main advantages of this method is that it allows the study of cellular processes at a greater specimen depth (500 $\mu \mathrm{m}$ to $1 \mathrm{~mm}$ ) compared with other traditional imaging techniques ${ }^{2}$. At the same time, the use of two low-energy photons generated by the two-photon laser minimizes the tissue photo-damage typically associated with the image acquisition process ${ }^{2}$. During the last decade, $2 \mathrm{P}$ IVM has been applied to study different types of cell-cell interactions in several disciplines ${ }^{3,4,5}$. These studies have been especially relevant to investigate immune cells, which are characterized by their high dynamism and the formation of prominent contacts following the signals generated by other cells and the environment. 2P-IVM has been also applied to study the interactions between pathogen and host ${ }^{6}$. Indeed, it has been previously shown that some pathogens can alter the type and duration of the contacts between immune cells, hampering, as a result, the immune response ${ }^{7}$.

The airway mucosa is the first site in which the immune response against airborne pathogens is generated ${ }^{8}$. Therefore, in vivo analysis of pathogen-host interactions in this tissue is critical to understand the initiation of the host defense mechanisms during infection. However, $2 \mathrm{P}$ IVM of the airways is challenging mainly due to the artifacts produced by the breathing cycle of the animal, which compromises the process of image acquisition. Recently, different surgical models have been described for imaging murine trachea ${ }^{9,10,11,12}$ and lungs ${ }^{13,14,15,16}$. Tracheal $2 \mathrm{P}$ IVM models represent an excellent set-up to visualize the initial phase of the immune reaction in the upper airways, while lung-alveoli 2P-IVM models are more suitable to study the late phase of infections. The developed lung models present a limitation associated with the presence of air-filled alveoli, which restrict the optical penetration of the laser and make the mucosal layer of the intrapulmonary airways inaccessible for in vivo imaging ${ }^{17}$. Conversely, the structure of the trachea, formed by a continuous epithelium, facilitates image acquisition.

Here, we present a protocol that includes a detailed description of the steps required to perform influenza infection, surgical preparation of the animals, and 2P-IVM of the trachea. In addition, we describe a specific experimental set-up for the visualization of neutrophils and dendritic cells (DC), two immune cell types that play an important role as mediators of the defense mechanism against influenza virus ${ }^{18,19}$. Finally, we describe 
a procedure to analyze neutrophil-DC interactions. These contacts have been shown to modulate DC activation and, subsequently, to affect the immune responses against pathogens ${ }^{20}$.

\section{Protocol}

All animal procedures involving mice were performed in accordance with the Swiss Federal Veterinary Office guidelines and animal protocols were approved by the local veterinarian authorities.

\section{Influenza Infection of CD11c-YFP Mice}

\section{Biosafety}

NOTE: The mouse adapted strain of influenza A/Puerto Rico/8/34 H1N1 (PR8) was grown in fertilized eggs, purified and titrated as previously described ${ }^{21}$. All the steps involving infected animals or biological samples were carried out under a biosafety cabinet according to biosafety level (BSL) 2 conditions.

1. Clean the biosafety cabinet with a $70 \%$ ethanol solution before and after the infection procedure.

2. Discard all waste materials produced during this procedure following proper WHO biosafety guidelines (http://www.who.int/csr/ resources/publications/biosafety/Biosafety7.pdf). Discard solid waste in autoclavable bins and contaminated liquids in plastic bags filled with $70 \%$ ethanol solution or medical disinfectant.

2. Influenza intranasal infection

NOTE: B6.Cg-Tg(Itgax-Venus)1Mnz/J (CD11C-YFP) $)^{22}$ on a C57BL/6J background were used in this study. Mice were maintained in the specific pathogen-free facility at the Institute for Research in Biomedicine.

1. Place a maximum of 5 age- and sex-matched (six to eight-week-old) CD11c-YFP mice per cage. Wait for at least two days for mice acclimation to housing conditions before the infection procedure.

2. Defrost PR8 stock and prepare the corresponding dilution using cold $1 \times$ Dulbecco's phosphate buffer saline modified without calcium chloride and magnesium chloride (PBS) to obtain a final concentration of 200 plaque forming units (PFU) in $30 \mu \mathrm{L}$. Keep virus dilution on ice during the whole procedure. NOTE: Titration of every batch of influenza virus prior to its use is recommended to ensure a precise infection dose.

3. Inject a dose of ketamine (100 mg/kg) and xylazine (10 mg/kg) intraperitoneally (i.p.) using a $26 \mathrm{G}$ needle $1 \mathrm{~mL}$ syringe.

4. Wait until the mouse is fully anesthetized (complete loss of both righting and pedal withdrawal reflex). Deep anesthesia is necessary for an optimal infection, since not-fully anesthetized mice will swallow or expel the virus inoculum, leading to variations in the infection dose.

5. Place the anesthetized mouse in a supine position. Collect $15 \mu \mathrm{L}$ of virus inoculum. Place the pipette tip close to the mouse left nostril and dispense the viral inoculum drop by drop. As drops must be inhaled, do not pipette them directly inside the nose cavity.

6. Wait 2-5 min and place the pipette tip containing the remaining $15 \mu \mathrm{L}$ of virus inoculum in the right nostril.

NOTE: To avoid suffocation, dispense small size drops in approximately $20 \mathrm{~s}$ intervals.

7. Check that the mouse is correctly breathing and place it in a cage in lateral decubitus. Monitor mouse breathing and anesthesia recovery (approximately $60 \mathrm{~min}$ after induction).

NOTE: It is possible to infect more than 1 mouse at the same time. In this case, administer viral inoculum in the left nostril of all mice in a sequence, wait for $2-5 \mathrm{~min}$ and then administer the virus in the right nostril. To avoid variability between individuals, infect no more than 3 mice at the same time.

8. Monitor animal health status and weight loss daily.

9. Euthanize mice according to the humane endpoint determined by the authority guidelines. The euthanasia method has to be approved by the animal experimentation authorities and must respect local ethical regulations. After two-photon experiments, euthanize mice by administration of an overdose of ketamine/xylazine followed by cervical dislocation. In all the other experiments, use $\mathrm{CO}_{2}$ inhalation as a method for euthanasia.

NOTE: To measure the viral titers of the infected trachea, $50 \%$ tissue culture infective dose (TCID50) assay or real time polymerase chain reaction (RT-PCR) assay can be performed as previously described ${ }^{23}$.

3. Evaluation of neutrophil recruitment in trachea by flow cytometry

NOTE: This part of the protocol is optional. It aims to evaluate neutrophil recruitment in tracheal mucosa following influenza infection.

1. Euthanize infected and uninfected control mice at day 3 post-infection (p.i.) by $\mathrm{CO}_{2}$ inhalation.

NOTE: In order to prevent tracheal damage, avoid using cervical dislocation to euthanize animals. Additionally, perfusion of euthanized mice is advisable to avoid contamination from blood neutrophils.

2. Spray the mouse neck with $70 \%$ ethanol solution and perform a skin incision using surgical scissors from the chest to the chin.

3. Separate the salivary glands using forceps and expose the trachea.

4. Dissect the muscles around the trachea using forceps and scissors.

NOTE: This step must be performed carefully since trachea could be easily damaged during the procedure.

5. Hold the trachea with forceps and carefully detach the esophagus by dissection.

6. Holding the intrathoracic part of the trachea with forceps, make an incision at the beginning of the bronchial tree. Then detach the trachea from the larynx and carefully remove any musculature left.

7. Place the organ in a $1.5 \mathrm{~mL}$ tube containing RPMI $1640+$ HEPES medium (RPMI) on ice.

8. Prepare the enzyme mixture for trachea digestion, containing 0.26 units $/ \mathrm{mL}$ of collagenase (I and II) and $0.2 \mathrm{mg} / \mathrm{mL}$ of DNase I in RPMI.

9. Place the dissected trachea in a 6-well plate containing $1 \mathrm{~mL}$ of enzyme mixture.

10. Cut the organ into small pieces with the help of forceps and scissors. Keep the plate on ice during this step.

11. Incubate at $37^{\circ} \mathrm{C}$ for $45 \mathrm{~min}$. During this time, shake the plate every $15 \mathrm{~min}$.

12. Stop the enzyme digestion by adding $1 \mathrm{~mL}$ of FACS washing buffer ( $2 \mathrm{mM}$ EDTA and $2 \%$ heat-inactivated filter-sterilized fetal bovine serum (FBS)) in PBS. 
13. Resuspend the solution with a $1 \mathrm{~mL}$ pipette to help to disassociate the partially undigested pieces of tissue.

14. Transfer the solution to another well by passing the content through a $40 \mu \mathrm{m}$ strainer. Then, gently smash the remaining pieces of the organ trapped on top of the strainer using a $2 \mathrm{~mL}$ syringe plunger.

NOTE: Smashing partially undigested pieces of trachea over the strainer is a critical step to obtain an optimal number of cells during flow cytometric analysis.

15. Wash the well and the strainer with FACS washing buffer and transfer the suspension in a $5 \mathrm{~mL}$ tube kept on ice.

16. Centrifuge the tubes at $166 \times \mathrm{g}$ for $5 \mathrm{~min}$ at $4{ }^{\circ} \mathrm{C}$. Discard the supernatant and resuspend the cells in $100 \mu \mathrm{L}$ of FACS washing buffer.

17. Proceed with antibody surface staining for flow cytometry. Briefly, block Fc receptors of the isolated cells using an antibody against CD16/32, followed by surface staining. To correctly identify neutrophils, the flow cytometry panel should contain the following antibodies: aLy6G, aCD11b, aCD45, as well as a viability dye to exclude dead cells.

18. Run the whole content of the samples on a flow cytometer and analyze the data.

1. To get an optimal number of immune cells, run the single cell suspension at a speed not higher than 3,000 events/s. This will reduce the number of events excluded during the acquisition. Using this protocol, it should be possible to obtain 1 to 2 million of cells per trachea.

\section{Isolation and Injection of Neutrophils}

NOTE: In this procedure, B6.129(ICR)-Tg(CAG-ECFP)CK6Nagy/J mice (CK6-ECFP) were used ${ }^{24}$. These animals express CFP in all cell types under the human $\beta$-actin promoter. Alternatively, it is also possible to use C57BL/6J mice to isolate cells and stain them according to the protocol described in step 2.6. The purification and manipulation of neutrophils may increase their activation status, potentially altering their migratory and functional properties.

1. Euthanize the animal by $\mathrm{CO}_{2}$ inhalation.

2. Remove both femurs and tibias and gently clean them using forceps.

3. Cut bone epiphyses and flush the bone marrow using a $1 \mathrm{~mL}$ syringe filled with sterile cold PBS, in a $50 \mathrm{~mL}$ tube kept on ice.

4. Resuspend the cells with an $18 \mathrm{G}$ needle and filter the cell suspension with a $40 \mu \mathrm{m}$ strainer. Wash once with PBS at $110 \times g$ for 5 min at 4 ${ }^{\circ} \mathrm{C}$ and resuspend cells in $2 \mathrm{~mL}$ of cold PBS.

5. Dilute $100 \%$ Percoll 9:1 in 10x PBS and prepare gradient solutions of percoll $72 \%, 64 \%$ and $52 \%$ using $1 \times$ PBS. Carefully layer $2 \mathrm{~mL}$ of each of the three gradients in a $15 \mathrm{~mL}$ tube, starting from the most concentrated one.

1. Add carefully $2 \mathrm{~mL}$ of the bone marrow cell suspension on top of the gradients and centrifuge at $1,100 \times \mathrm{g}$ for $30 \mathrm{~min}$ at room temperature (RT) without acceleration and brake.

6. Carefully remove the band at the interface between $64 \%$ and $72 \%$ and wash it once at $200 \times g$ for 5 min at $4{ }^{\circ} \mathrm{C}$ with cold PBS. Resuspend cells in a volume of $100 \mu \mathrm{L}$ of PBS and keep them on ice. The purity of the neutrophils is expected to be higher than $90 \%$.

7. Optionally, label neutrophils with a cell proliferation kit using the manufacturer's protocol. Add the dye to the cell suspension to a final concentration of $10 \mu \mathrm{M}$ in a volume of $1 \mathrm{~mL}$, incubate at $37^{\circ} \mathrm{C}$ for $30 \mathrm{~min}$ and wash $(166 \times \mathrm{g}, 5 \mathrm{~min})$.

8. Estimate cell concentration using a hemocytometer and inject intravenously $5 \times 10^{6}$ cells in a maximum volume of $100 \mu \mathrm{L}$ in the previously infected CD11c-YFP ${ }^{+}$mice using a $26 \mathrm{G}$ needle $1 \mathrm{~mL}$ syringe, $12 \mathrm{~h}$ before imaging.

\section{Preparing the Mouse for Imaging}

\section{Anesthesia}

1. Anesthetize infected CD11c-YFP ${ }^{+}$mice at day 3 p.i. according to step 1.2.3.

2. Once mice are deeply anesthetized, prepare a catheter for anesthesia re-dosing.

1. Remove a $30 \mathrm{G}$ needle from a syringe using forceps.

2. Insert the needle to a $20 \mathrm{~cm}$ piece of PE-10 medical silicon tubing.

3. Insert a $30 \mathrm{G}$ needle syringe filled with ketamine/xylazine mixture on the other side of the tube.

4. Remove the air inside of the tube.

3. Prepare a $20 \mathrm{G}$ catheter connected to a tube from an oxygen insufflating machine to maintain automated ventilation (Figure 1Ai). Set it on a breath ratio of 130 beats per minute (b.p.m.) with a tidal volume of $0.2 \mathrm{~mL}$ using a $100 \%$ oxygen gas supply.

2. Prepare the mouse for surgery

1. Keep the mouse on a specific customized surgical board (Figure 1Aii-iii) over a heated plate or a heated surgical bench set at $37{ }^{\circ} \mathrm{C}$ for all the time of the surgery.

2. Shave the hair from the neck of the mouse using an electric razor and a depilatory cream (Figure 1Bi).

3. Place the mouse above the plastic mouse positional (Figure 1Aii-a), keeping the animal head outside of the positional (Figure 1Bii) to create an angle that facilitates the intubation of the trachea.

4. Fix the forelimbs, the paws, and the tail with surgical tape, moisten mouse eyes with a vitamin A enriched gel and disinfect the mouse neck (Figure 1Bii).

\section{Surgery}

1. Prepare properly autoclaved items, namely, scissors, microsurgical scissors, and forceps.

2. Perform a small incision on the long medial axis of the neck, approximately $1 \mathrm{~cm}$ long, in the middle area between the upper chest and the line passing through the lower point of the mandible (Figure 1Biii).

3. Move laterally the skin patches and the salivary glands and visualize the trachea, covered by the muscles. Then, dissect the tracheal muscles carefully using the forceps (Figure 1Biii).

4. Intubate the mouse with a catheter and start artificial ventilation (Figure 1Biv). 
NOTE: The catheter is composed of an external plastic part, which protects the tracheal mucosa, and an inner iron needle. The presence of the needle represents the ideal solution to extend and stabilize the trachea and to regulate its exposition. Artificial ventilation through the catheter will guarantee proper breathing of the mouse.

5. Immediately start the artificial ventilation to monitor mouse breathing.

6. Fix the catheter height and orientation using a surgical hook (Figure 1Bv) connected to the specific rod on the surgical board (Figure 1Aii-b). Expose the trachea at the same height of the chin.

7. Encircle the trachea with petroleum jelly and cover it with a few drops of pre-heated PBS to guarantee proper hydration to the organ (Figure 1Bvi).

8. Mount the coverslip on top of the preparation. For this step, glue a coverslip on a metal holder (Figure 1Bvii), which will be screwed to the $\mathrm{XYZ}$ translator (Figure 1Aii-c). Adjust the $\mathrm{XYZ}$ translator to place the coverslip on top of the surgical preparation.

9. Place the catheter for the administration of the anesthesia intraperitoneally (Figure 1Bviii).

10. Inject $50 \%$ of the initial dose of ketamine/xylazine mixture every $30 \mathrm{~min}$.

NOTE: Re-dosing from $50 \%$ to $25 \%$ of the initial ketamine/xylazine mixture is a safe and valid alternative ${ }^{25}$. However, since this protocol is terminal and a strict immobilization of the animal is required during the whole procedure, use higher dosages to maintain a deep surgical plane of anesthesia.

\section{In Vivo Time-lapse Imaging}

NOTE: Image acquisition was performed with an upright two-photon microscope, equipped with two Ti:Sa lasers, temperature-controlled incubation chamber, and a 25X/NA 1.1 water immersion objective. The photomultipliers (PMT) used for image acquisition were either hybrid detectors or high sensitivity GaAsP.

1. Place the surgical board with the anesthetized mouse inside the microscope incubation chamber (pre-heated at $37-38{ }^{\circ} \mathrm{C}$ ) and add a drop of water on the coverslip.

2. Center and find focus on the tracheal tissue.

3. Set the scanning frequency to $800 \mathrm{~Hz}$, with $520 \times 520$ pixels, a field of view of $440 \times 440 \mu \mathrm{m}^{2}$ and line average 1 .

1. Tune Ti:Sa laser to $830 \mathrm{~nm}$ to excite second harmonic generation (SHG) from collagen, with an indicative power at source of $150 \mathrm{~mW}$. Tune the second Ti:Sa laser at $920 \mathrm{~nm}$ with $94 \mathrm{~mW}$ at source, to excite both CFP and YFP.

2. Set-up the 3D and timelapse acquisition mode with simultaneous excitation NOTE: Keep the laser powers as low as possible to minimize photobleaching and phototoxicity.

4. Record fluorescence using two channels in non-descanned mode, with a master dichroic mirror at $560 \mathrm{~nm}$. In the set-up used in this protocol, a second dichroic mirror split the signal at $495 \mathrm{~nm}$ to separate channel 1 (emission filter 475/50) from channel 2 (emission filter 525/50) (Figure 2A). This configuration collects SHG light from collagen in the first channel, while CFP emission is collected in both channels 1 and 2 and YFP is collected only in channel 2.

5. Define a range $50 \mu \mathrm{m}$ along the $Z$ axis, with a step size of $3 \mu \mathrm{m}$ (voxel size $0.86 \mu \mathrm{m} \times 3 \mu \mathrm{m}$ ). Record images every $30 \mathrm{~s}$ for a total duration of $30 \mathrm{~min}$.

6. If desired, acquire multiple regions. Before running more acquisitions, check vital signs and reinject anesthesia through the catheter if needed (see step 3.3.10).

7. At the end of the imaging process, euthanize the mouse through ketamine/xylazine overdose followed by cervical dislocation.

\section{Image Processing and Quantitative Analysis of Neutrophil-DC Motility and Interaction}

NOTE: In this protocol, a specialized imaging software was used for analyzing the microscopy data.

1. After $4 \mathrm{D}$ image acquisition is completed, transfer the files (data and metadata) on a workstation with sufficient computational resources (suggested minimum system requirements: 32 GB RAM, fast solid-state-drives, recent CPU, dedicated GPU based on a massively parallel architecture).

2. Open the files in the imaging software.

3. Play the video and ensure that the cells of interest are clearly visible and that imaging artifacts are absent. NOTE: To this end, verify that both the movement of the sample and the brightness variation are sufficiently confined. Indeed, these represent challenges for the automatic analysis ${ }^{26}$. If the movement of the sample between adjacent frames is excessive, apply a drift correction method, using for instance the SHG channel as a fixed reference. This allows to better measure the movement of the cells rather than the movement of the sample. Additionally, in the presence of bright background or debris, prune the reconstructed surfaces using volume as a selection parameter.

4. Generate a co-localization channel specific for $\mathrm{CFP}^{+}$cells, in order to separate the signal between CFP and the collagen (SHG). To this end, denote a gating polygon (Figure $\mathbf{2 B i}$ ) that selects only the voxels having a positive intensity both in the green channel and in the blue channel.

NOTE: This procedure may vary according to the filter set of the microscope and the staining of the cells. It can be achieved by selecting only voxels with sufficient intensity in both the green and the blue channels. Amongst the available tools, the "coloc" functionality can be used to automatically compute the colocalization channel based on intensity thresholding. Additionally, methods based on machine learning can be used for this step with the supervision of an expert ${ }^{27}$.

5. Detect and track CFP ${ }^{+}$cells, using automatic surface reconstruction and tracking (surface tool) over the appropriate co-localization channel. NOTE: Manual curation of eventual tracking errors and exclusion of tracks with a duration shorter than a defined threshold (i.e., $150 \mathrm{~s}$ ) may be required.

6. Generate a co-localization channel specific for CD11c-YFP ${ }^{+}$cells, in order to separate CFP and YFP signals. To this end, denote a gating polygon (Figure 2Bii) that selects only the voxels having a positive intensity in the green channel but a low intensity in the blue channel. 
NOTE: Representative micrographs showing the signals from channel 1, channel 2, the co-localization channel for CFP, the co-localization channel for YFP, and the combination of all channels can be found in Figure 2Biii.

7. Reconstruct the surface of the CD11c-YFP ${ }^{+}$cells and track their position over time (surface tool).

NOTE: Manual correction of eventual tracking errors is not required for this step. Indeed, due to the complex spatio-temporal dynamics of DC, reconstructing their precise surface from 2P-IVM data is a challenging task that cannot be achieved with available segmentation software. Amongst the reasons that make this task challenging, thin protrusions and brightness variations do not allow for the usage of certain image-processing techniques, such as smoothing filters or static thresholding. Moreover, in a network of DC, it is difficult to separate single cells based only on their appearance in 2P-IVM data. For these reasons, rather than attempting an accurate segmentation by tuning software parameters, we propose to reconstruct non-accurate surfaces of DC. Then, handle the possible errors by means of robust metrics as described in 5.8 .

8. Measure cell migration.

1. Export the classical migration measures for both $\mathrm{CFP}^{+}$and CD11c-YFP ${ }^{+}$cells. Amongst these, the "track speed mean" indicates the average migratory velocity of the cells while the "track straightness" indicates the directionality of the cells. These measures can be exported as a spreadsheet file from the imaging software.

2. In the exported spreadsheet files, compute the "corrected track straightness" (also referred to as corrected confinement ratio) for both $\mathrm{CFP}^{+}$and CD11c-YFP ${ }^{+}$cells, which is defined as "track straightness" multiplied by the square root of "track duration" divided by the square root of the video duration. This measure is more robust than "track straightness" in the presence of short tracks ${ }^{28}$, originating for instance from tracking errors.

NOTE: only videos of the same length can be compared with this measure.

\section{Measure cell interaction.}

1. Define a contact between a CFP ${ }^{+}$cell and a CD11c-YFP ${ }^{+}$cell if their distance (closest voxels) is less or equal than a threshold (i.e., 2 $\mu \mathrm{m})$.

NOTE: This threshold should be sufficiently strict to detect a contact only when cells are in close proximity. However, we encourage to keep this threshold greater than 0 , ideally $\mathrm{N}$ times bigger than the voxel radius $(\mathrm{N}>2)$, because border smoothing can make the reconstruction of cells smaller than the actual cell size.

2. Count the number and the duration of contacts between $\mathrm{CFP}^{+}$and CD11c-YFP ${ }^{+}$cells. In the imaging software this can be done for instance by executing a "kiss and run" plug in. These measures can be exported as a spreadsheet file from the statistics tab.

10. Import the previously computed measures in a statistical software, generate the plots and perform statistical tests.

\section{Representative Results}

In this work, we described a detailed protocol to study in vivo the motility and the interactions between neutrophils and DC during influenza infection in murine trachea (Figure 3A). To this purpose, we isolated CFP ${ }^{+}$neutrophils (92\% purity; Figure 3B) from CK6-ECFP mice and we adoptively transferred them into a CD11C-YFP mouse infected with influenza. After that, we performed 2P-IVM of the trachea at day 3 p.i. At this time point we observed a clear recruitment of neutrophils in the infected area, as shown by flow cytometric analysis (Figure 3C). The 2PIVM protocol requires the use of a specific surgical board and an oxygen supplier for rodents (Figure 1A). Supplying oxygen through a cannula inserted in the trachea helped the animal breathing, facilitated the exposition of the trachea, and controlled the organ movement associated with breathing (Figure 1B). Following this experimental set-up, we acquired stable 4D images in vivo in the infected trachea during a period of 30 min (Figure 3D, Movie 1).

The analysis of the acquired 4D images through specialized imaging software allowed to measure the migration of cells and to quantify the spatio-temporal dynamics of neutrophils and DC. Regarding cell motility, we observed significant differences between the movement of DC and the recruited neutrophils, which showed a significantly faster speed than the latter (Figure 4A). This result confirms the dynamic nature of neutrophils, previously described as highly motile cells capable of migrating towards a chemoattractant source ${ }^{29}$. Regarding directionality, we concluded that complex morphology of the DC yielded frequent errors in cell tracking, which in turn produced tracks with decreased duration and increased variance of the measured directional behavior (Figure 4B). For this reason, we computed a robust metric that is able to measure directionality by considering track duration. Using this metric, we observed a significant difference in the directionality of neutrophils vs DC (Figure 4C).

Additionally, the computation of the distance between neutrophils and DC allowed to detect and to analyze their contacts over time. In this experimental model, we observed some neutrophils that formed multiple-brief contacts with DC and others that did not form any contact during the imaged period (Figure 4D). Moreover, the study of the average trend of the distance between neutrophils and DC over time allowed us to study the overall positioning of the studied cells (Figure 4E), while the investigation of the trend in specific cells allowed to characterize the behavior of each single-cell (Figure 4F, Movie 2). 


\section{A Custom-built imaging platform and oxygen supplier}
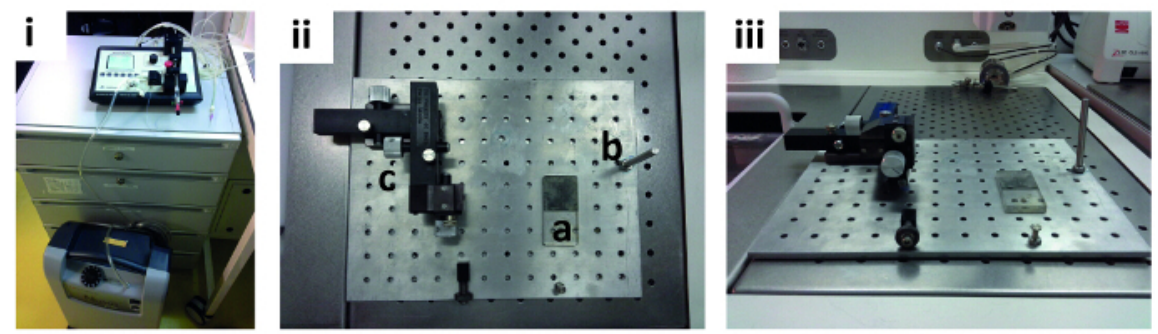

\section{B Steps for preparing the mouse for imaging}
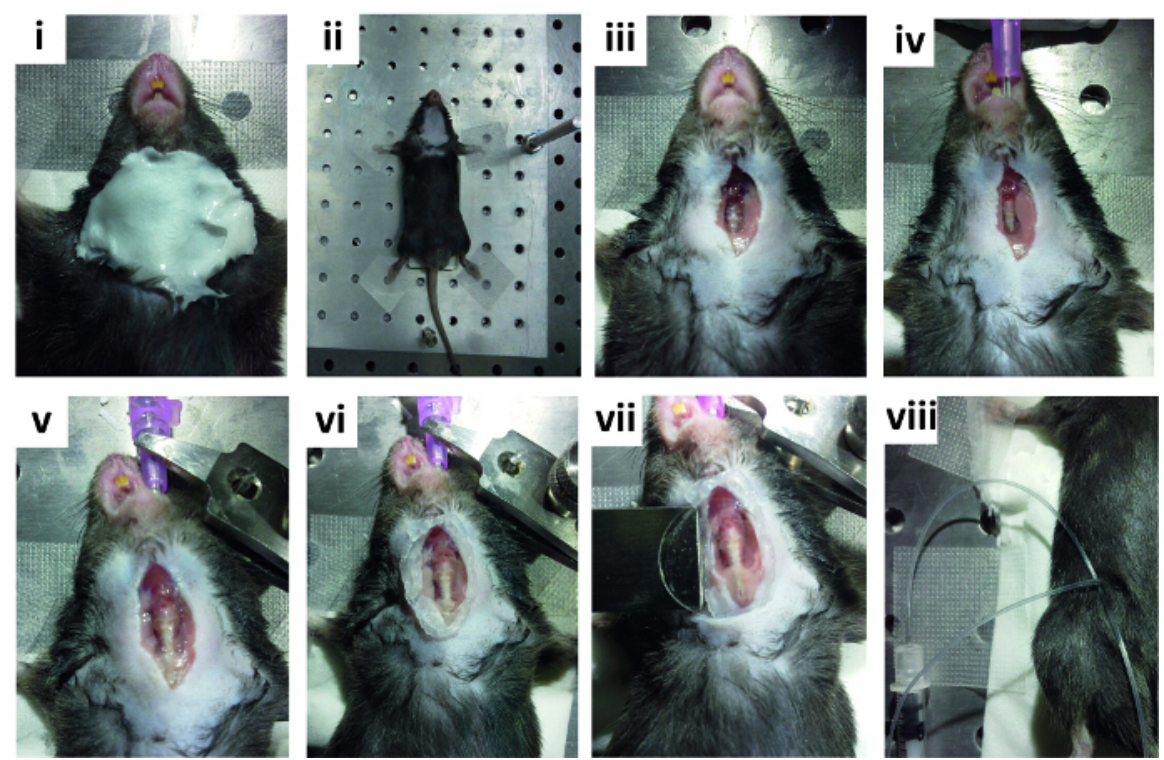

Figure 1: Equipment and steps for 2P-IVM of murine trachea. (Ai) The portable animal anesthesia system in charge of the automated ventilation is connected to a pump that supplies oxygen to the mouse. Front view (Aii) and side view (Aiii) of the custom-made surgical board used for the tracheal model. The board is composed of a metal stage with a plastic mouse positional (Aii-a), a rod for holding a movable clamp (Aii-b), and a fine tunable XYZ translator (Aii-c). (B) Sequential steps of the tracheal surgical model: (Bi) hair removal of the surgical area, (Bii) positioning of the anesthetized mouse in the surgical board, (Biii) surgical exposition of the trachea, (Biv) intubation with a catheter with artificial ventilation, (Bv) fixation of the catheter, (Bvi) addition of PBS to the exposed trachea, (Bvii) mounting of the coverslip, and (Bviii) placement of a catheter with anesthesia. Please click here to view a larger version of this figure. 


\section{A PMT and filter set-up of 2PM}

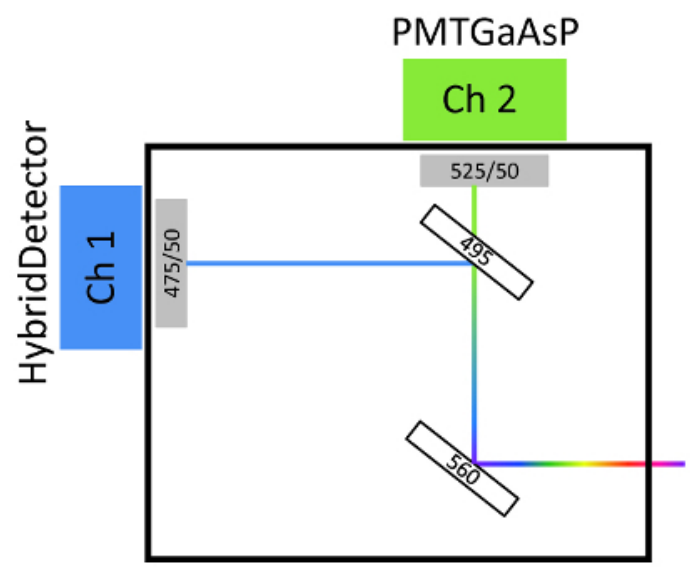

\section{B Generation of co-localization channels}

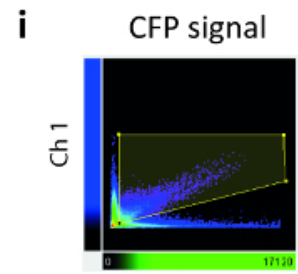

Ch 2

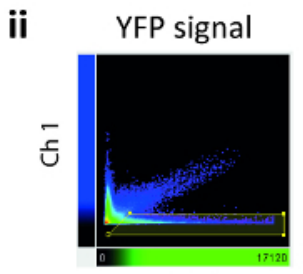

Ch 2

iii

Ch 1

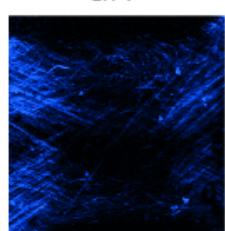

Ch 2

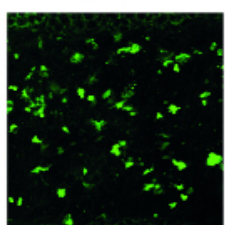

CFP signal

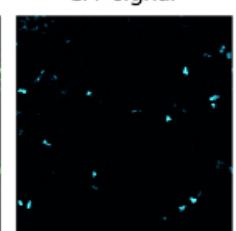

YFP signal

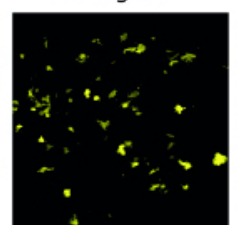

Ch $1+$ Ch 2+CFP+YFP

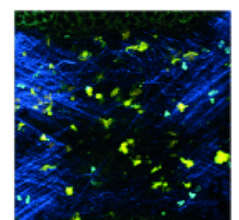

Figure 2: Fluorescent signal detection during 2P-IVM. (A) Schematic representation of the microscope detection filter set-up and corresponding channels. Dichroic mirror at $560 \mathrm{~nm}$ separates blue/green from red/far red emissions. Additional dichroic mirror at $495 \mathrm{~nm}$ is used to further recognize the different subregions of the emission spectrum. Channel 1 employs a hybrid detector (emission filter 475/50), while channel 2 uses high sensitivity GaAsP PMT (emission filter 525/50). (B) Representative scatter dot plots of 2P signals showing the gating strategy for the generation of the colocalization channels for the identification of signal coming from the CFP (Bi) and the YFP (Bii) fluorophores. (Biii) Representative micrographs showing the specific signals from channel 1 (Ch 1, dark blue), channel 2 (Ch 2, green), the co-localization channel for CFP (light blue), the co-localization channel for YFP (yellow), and the combination of all channels (Ch $1+C h 2+C F P+Y F P)$. Please click here to view a larger version of this figure. 


\section{A Experimental outline}

$$
\text { Day } 0 \text { p.i. Day } 2 \text { p.i. Day } 3 \text { p.i. }
$$

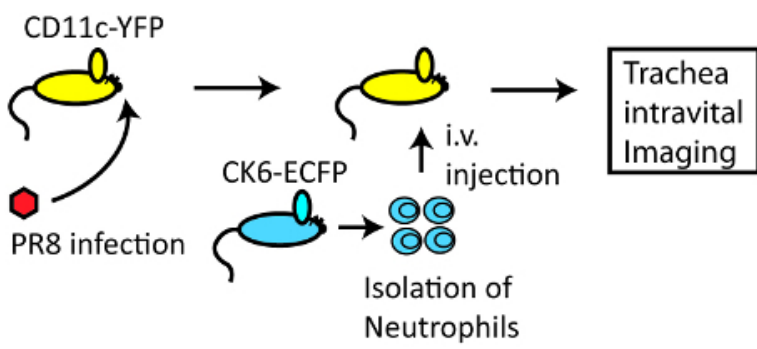

\section{B Purity of isolated} Neutrophils

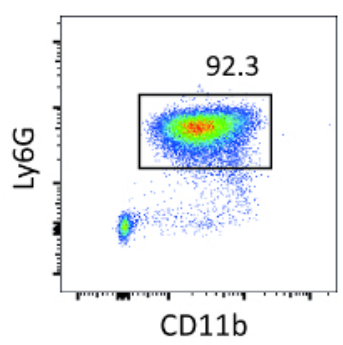

C Neutrophils in trachea at day 3 p.i.

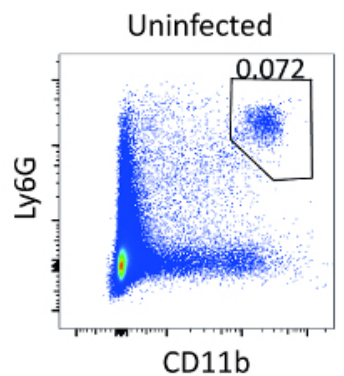

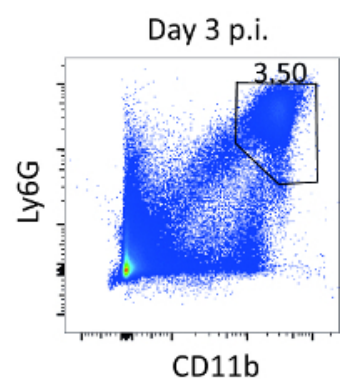

D 2P-IVM imaging of murine trachea

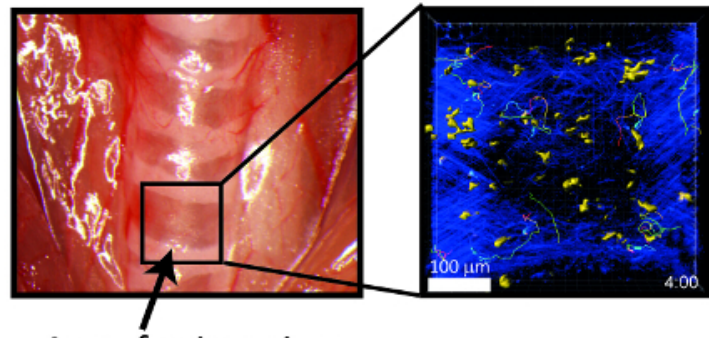

DC

Neutrophils

SHG

Area for imaging

Figure 3: Intravital 4D imaging of neutrophils and DC in an influenza infected trachea. (A) Schematic outline of the protocol. (B) Representative flow cytometric scatterplot showing the percentage of neutrophils with respect to the total CD45+ cells in a cell suspension isolated from murine bone marrow using the Percoll gradient method. (C) Representative flow cytometric scatterplots showing an increase in the frequency of neutrophils in tracheas from uninfected mice compared to mice infected with influenza virus at day 3 p.i. (D) (Left panel) Anatomical image of a murine trachea showing the area selected for image acquisition. (Right panel) Representative 3D projection of a 2P-IVM micrograph showing the surface reconstruction of neutrophils (light blue) and DC (yellow) together with their tracks at day 3 p.i. SHG signal is shown in dark blue. Scale bar $=100 \mu \mathrm{m}$. Please click here to view a larger version of this figure. 
A Track Speed Mean

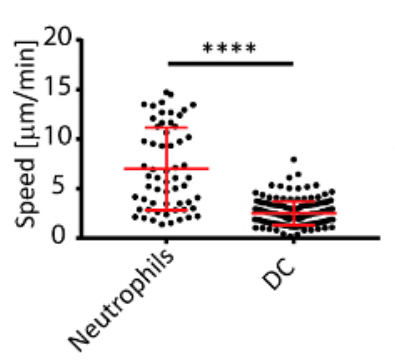

B Track Straightness

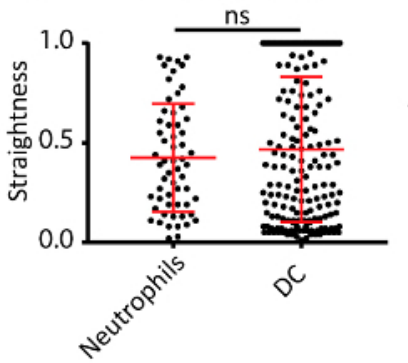

C Corrected Track Straightness

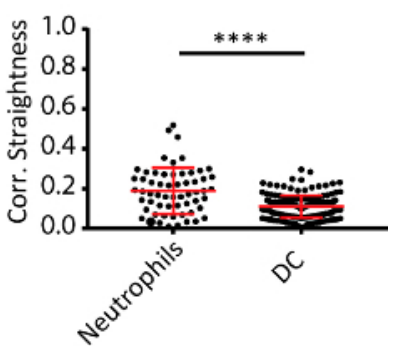

D Characterization of neutrophil-DC contacts
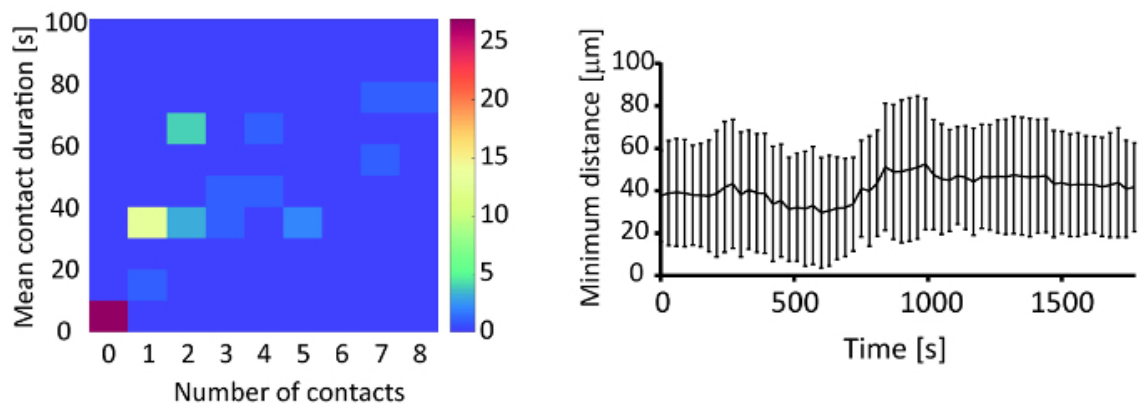

F

Distance of a single neutrophil to the closest DC
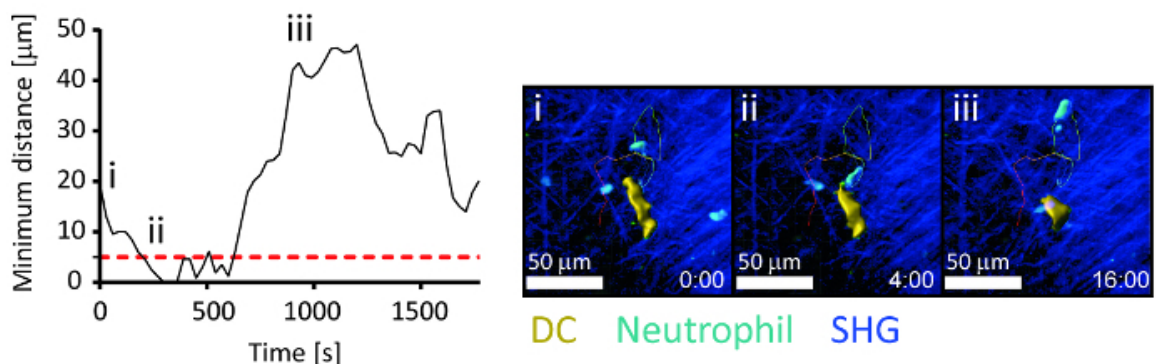

Figure 4: Characterization of neutrophil and DC migration and interaction dynamics in influenza infected trachea. Representative plots showing the track speed mean (A), track straightness (B), and corrected track straightness (C), as defined by Beltman and colleagues (2009) ${ }^{28}$, of neutrophils and DC in trachea at day 3 p.i. with influenza virus. The corrected track straightness measurement exhibits robustness to tracking errors. (D) 2D histogram showing the frequency of neutrophil according to their number of contacts with DC and the mean contact duration. (E) Average distance of neutrophils to the closest DC during the duration of the movie. (F) (Left) Analysis of the distance of a representative neutrophil to the closest DC in time. The dotted red line indicates the distance threshold to consider that a neutrophil established a contact with a DC. (Right i-iii) Micrographs acquired at different time points representing the migration of a neutrophil (light blue) towards a DC (yellow). Cell tracks are shown as a multicolored line that changes color from blue to red to represent time. SHG signal from fibrillary collagen is shown in dark blue. Scale bar $=50 \mu \mathrm{m}$. In all figures, the presented data are representative of at least three independent experiments. Results are given as mean \pm SD. Statistics by Welch's test. ns $p>0.05$; ${ }^{* * *} p<0.0001$. Please click here to view a larger version of this figure. 


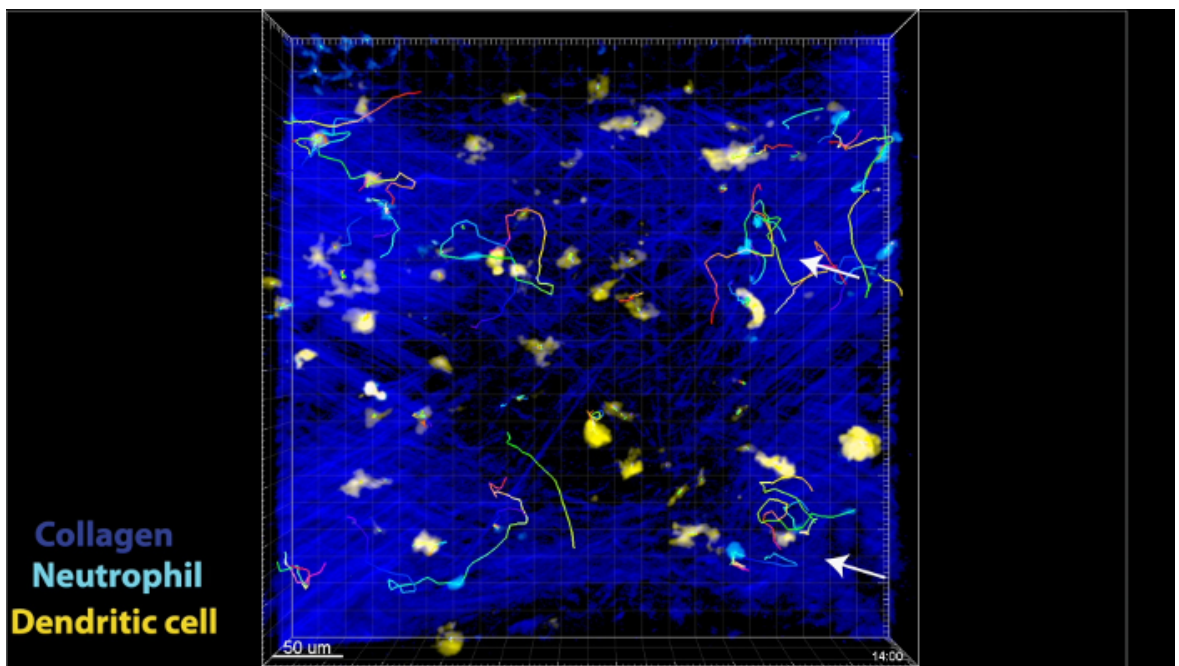

Movie 1: Neutrophils and DC dynamics in trachea during influenza infection. 30 min time-lapse 3D image showing interaction dynamics between neutrophils (light blue) and DC (yellow) as well as their respective tracks with respect to the collagen network (dark blue) of the trachea. Representative neutrophil-DC interactions are indicated by white arrows. Cell tracks are shown as a multicolored line that changes color from blue to red to represent time. Scale bar $=50 \mu \mathrm{m}$. Please click here to view this video. (Right-click to download.)

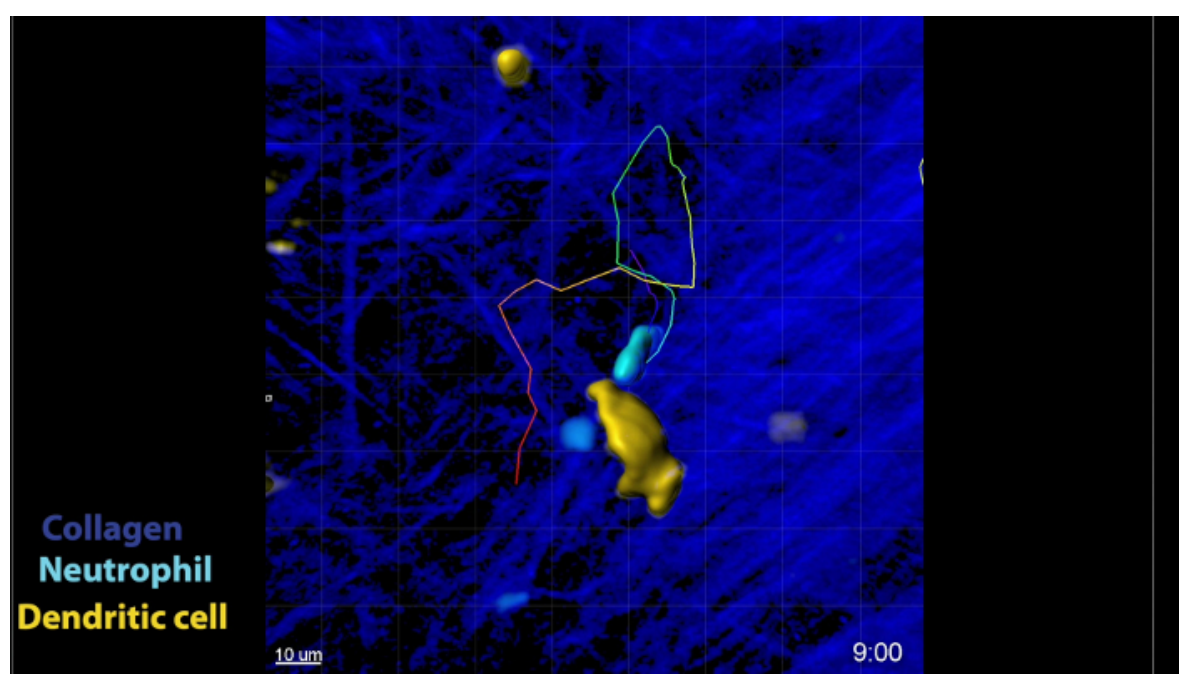

Movie 2: Representative short-term neutrophil-DC interaction in the trachea during influenza infection. 30 min time-lapse 3D image showing a representative interaction between a neutrophil (light blue) and a DC (yellow) and their respective tracks. Cell tracks are shown as a multicolored line that changes color from blue to red to represent time. SHG signal from collagen is shown in dark blue. Scale bar $=10 \mu \mathrm{m}$. Please click here to view this video. (Right-click to download.)

\section{Discussion}

This work presents a detailed protocol for the generation of 4D images showing the migration of adoptively transferred neutrophils and their interactions with DC during an influenza infection in the mouse trachea. The described 2P-IVM model will be relevant to study immune cell dynamics during an infection in the airways.

Recently, several models based on the visualization of cell dynamics in the airways have been developed ${ }^{9,10,11,12,13,14,15,16}$. However, in vivo imaging of the lung is still challenging, considering the anatomic position of this organ and the technical difficulties to minimize the movement during the breathing cycle $e^{30}$. To overcome these problems, some authors have proposed the use of a custom-built circular suction chamber, which needs to be surgically inserted in the thorax ${ }^{13,14}$. However, this procedure requires an invasive intervention that could compromise the results, especially in those studies that have focused on the investigation of the inflammatory response. Furthermore, lung surgical models present a limitation for deep tissue imaging due to the light refraction originated by the air in the alveoli ${ }^{17}$. Conversely, different tracheal models have been recently employed to study cell dynamics in the airway epithelium. Imaging of this organ presents clear advantages compared to lung, such as the relatively simple surgery required to expose and immobilize the organ, as well as the higher accessibility to the tracheal epithelium. The proposed tracheal model is also relevant to investigate the initiation of the response against airway pathogens, such as influenza virus, since the trachea is one of the first sites of viral replication during the course of an influenza infection ${ }^{8}$.

Interestingly, a study showing an alternative intubation-free method for imaging the trachea has been recently published ${ }^{12}$. This method is characterized by a decreased inflammation and shows clear advantages in studies where the mucociliarity function of the epithelial cells needs 
to be preserved. However, this method does not guarantee sufficient stability and the acquisition of brighter signals necessary to study cellcell contacts in a range of a few $\mu \mathrm{m}$. Conversely, the method presented in the current protocol provides better immobilization of the organ thanks to the intubation, and allows the detection of stronger fluorescence signals as a result of the shorter distance between the organ and the coverslip ${ }^{12}$.

Accomplishing tissue immobilization during in vivo 2P-IVM image acquisition is the most critical step to generate optimal data. Some crucial measures that contribute to the stability of the presented method include: an appropriate mouse anesthesia; a correct mouse intubation; and a surgical exposition of the trachea that allows an easy access to the organ by the coverslip. Additionally, imaging the right number of cells (ideally 30 cells per field of view) will strengthen the obtained results. The recruitment of the optimal number of cells will depend to a large extent on the viral infection dose, which is very much influenced by the proper administration of the viral inoculum.

Another critical step of the protocol is the surgical exposition of the trachea. Different measures can be adopted to minimize the damage caused to the organ during surgery. For example, the trachea should not be directly touched with surgical tools. Instead, it should be exposed by manipulating only the surrounding tissues (skin, salivary glands, and muscles). If strictly necessary, the trachea should be handled using unsharpened items. Additionally, efforts should be done to avoid blood vessel damage. Finally, to prevent organ dehydration, it is also important to cover it with PBS immediately after surgery.

Despite the unique advantages of this method over the previously described methods for the visualization of the immune cell interactions in the tracheal mucosa, the use of this model presents some limitations. As described above, the presence of inflammation associated with the tracheal surgery might represent a drawback when studying immune responses. To overcome this limitation, it is possible to administer anti-inflammatory drugs prior to the initiation of the procedure. Another limitation of this model is related to the presence of a strong autofluorescence signal existing in the airways, which is mainly generated by the resident cells and the mucus layer. This non-specific fluorescence creates artefacts that might hinder the analysis. Furthermore, misleading calculation of the track straightness parameter might be generated when comparing cell tracks of different durations and when tracking errors introduce tracks of short duration ${ }^{26}$. To overcome this problem, we applied a penalty coefficient to correct track straightness. Such correction is intended to minimize the effect of miss-tracking in the results ${ }^{28}$.

A crucial aspect of 2P-IVM experiments is the possibility to re-use mice that have undergone surgery and imaging. The in vivo imaging protocol described here does not require animal euthanasia or organ collection, thus leaving the possibility to recover and re-use mice after surgery for other procedures. Using a single mouse, for example, to perform trachea imaging at different time points could dramatically decrease the number of total animals needed in an experiment, supporting the animal reduction principle. Moreover, it could also reduce inter-individual variability. However, animal recovery and re-use must follow animal welfare standards that includes the administration of proper analgesic drugs and antibiotics to the animals during recovery time. All these procedures must be included in the animal experimentation protocol and approved by the local veterinarian authorities.

The described protocol can be easily adapted to the study of other immune cell types. For example, isolation and injection of (fluorescent or stained) pathogen-specific T cells might be used to study T cell activation dynamics ${ }^{31}$ as well as their interaction with other cells such as tracheal DC. In a similar way, the visualization of blood or lymphatic vessels could represent an interesting approach to study the recruitment of inflammatory cells into the tracheal tissue during the course of infection. Moreover, 2P-IVM of the trachea could also be applied to study the dynamics of the immune response to other airborne pathogens. Therefore, the use of transgenic fluorescent airborne pathogens, such as Streptococcus pneumoniae ${ }^{32}$, will create new opportunities to study their interactions with the immune system. Although this procedure focuses on measuring the dynamics of immune cells during infection, it could be also applied to different fields including cancer, asthma, or woundhealing.

\section{Disclosures}

The authors have nothing to disclose.

\section{Acknowledgements}

This work was supported by the Swiss National Foundation (SNF) grants (176124, 145038, and 148183), the European Commission Marie Curie Reintegration Grant (612742), and the SystemsX.ch for a grant to D.U.P. (2013/124).

1. Helmchen, F., Denk, W. Deep tissue two-photon microscopy. Nature Methods. 2 (12), 932-940 (2005).

2. Zipfel, W.R., Williams, R.M., Webb, W.W. Nonlinear magic: multiphoton microscopy in the biosciences. Nature biotechnology. 21 (11), 1369-77 (2003).

3. Fein, M.R., Egeblad, M. Caught in the act: revealing the metastatic process by live imaging. Disease Models \& Mechanisms. 6 (3), $580-593$ (2013).

4. Dombeck, D.A., Harvey, C.D., Tian, L., Looger, L.L., Tank, D.W. Functional imaging of hippocampal place cells at cellular resolution during virtual navigation. Nature Neuroscience. 13 (11), 1433-1440 (2010).

5. Cahalan, M.D., Parker, I. Choreography of cell motility and interaction dynamics imaged by two-photon microscopy in lymphoid organs Annual review of immunology. 26, 585-626 (2008).

6. Germain, R.N., Robey, E.A., Cahalan, M.D. A Decade of Imaging Cellular Motility and Interaction Dynamics in the Immune System. Science. 336 (6089), 1676-1681 (2012).

7. Coombes, J.L., Robey, E.A. Dynamic imaging of host-pathogen interactions in vivo. Nature Reviews Immunology. 10 (5), $353-364$ (2010).

8. Pulendran, B., Maddur, M.S. Innate Immune Sensing and Response to Influenza. Life Science Journal. 6 (4), 23-71 (2014).

9. Lim, K. et al. Neutrophil trails guide influenza- specific CD8 + T cells in the airways. Science. 349 (6252) (2015). 
10. Kim, J.K. et al. In vivo imaging of tracheal epithelial cells in mice during airway regeneration. American journal of respiratory cell and molecular biology. 47 (6), 864-8 (2012).

11. Kretschmer, S. et al. Autofluorescence multiphoton microscopy for visualization of tissue morphology and cellular dynamics in murine and human airways. Laboratory investigation; a journal of technical methods and pathology. 96 (8), 918-31 (2016).

12. Veres, T.Z. et al. Intubation-free in vivo imaging of the tracheal mucosa using two-photon microscopy. Scientific Reports. 7 (1), 694 (2017).

13. Looney, M.R. et al. Stabilized imaging of immune surveillance in the mouse lung. Nature methods. 8 (1), 91-6 (2011).

14. Thornton, E.E., Krummel, M.F., Looney, M.R. Live Imaging of the Lung. Current Protocols in Cytometry. 60 (1) (2012)

15. Tabuchi, A., Mertens, M., Kuppe, H., Pries, A.R., Kuebler, W.M. Intravital microscopy of the murine pulmonary microcirculation. Journal of Applied Physiology. 104 (2), 338-346 (2008).

16. Fiole, D. et al. Two-photon intravital imaging of lungs during anthrax infection reveals long-lasting macrophage-dendritic cell contacts. Infection and immunity. 82 (2), 864-72 (2014).

17. Secklehner, J., Celso, C. Lo, Carlin, L.M. Intravital microscopy in historic and contemporary immunology. Immunology and Cell Biology. 95 (6), 506-513 (2017).

18. Lambrecht, B.N., Hammad, H. Lung Dendritic Cells in Respiratory Viral Infection and Asthma: From Protection to Immunopathology. Annual Review of Immunology. 30 (1), 243-270 (2012).

19. Camp, J. V., Jonsson, C.B. A role for neutrophils in viral respiratory disease. Frontiers in Immunology. 8 (MAY) (2017).

20. van Gisbergen, K.P.J.M., Sanchez-Hernandez, M., Geijtenbeek, T.B.H., van Kooyk, Y. Neutrophils mediate immune modulation of dendritic cells through glycosylation-dependent interactions between Mac-1 and DC-SIGN. The Journal of experimental medicine. 201 (8), $1281-92$ (2005).

21. Gonzalez, S.F. et al. Capture of influenza by medullary dendritic cells via SIGN-R1 is essential for humoral immunity in draining lymph nodes. Nature Immunology. 11 (5), 427-434 (2010).

22. Lindquist, R.L. et al. Visualizing dendritic cell networks in vivo. Nature immunology. 5 (12), 1243-1250 (2004).

23. Li, H. et al. Human Vү9Vס2-T cells efficiently kill influenza virus-infected lung alveolar epithelial cells. Cellular and Molecular Immunology. 10 (2), 159-164 (2013).

24. Tran Cao, H.S. et al. Development of the transgenic cyan fluorescent protein (CFP)-expressing nude mouse for "technicolor" cancer imaging Journal of Cellular Biochemistry. 107 (2), 328-334 (2009).

25. Jaber, S.M. et al. Dose regimens, variability, and complications associated with using repeat-bolus dosing to extend a surgical plane of anesthesia in laboratory mice. Journal of the American Association for Laboratory Animal Science: JAALAS. 53 (6), 684-91 (2014).

26. Pizzagalli, D.U. et al. Leukocyte Tracking Database, a collection of immune cell tracks from intravital 2-photon microscopy videos. Scientific Data. In press (2018)

27. Sommer, C., Straehle, C., Kothe, U., Hamprecht, F.A. Ilastik: Interactive learning and segmentation toolkit. 2011 IEEE International Symposium on Biomedical Imaging: From Nano to Macro. 230-233 (2011).

28. Beltman, J.B., Marée, A.F.M., De Boer, R.J. Analysing immune cell migration. Nature Reviews Immunology. 9 (11), $789-798$ (2009).

29. Keller, H.U. Motility, cell shape, and locomotion of neutrophil granulocytes. Cell motility. 3 (1), 47-60 (1983).

30. Sumen, C., Mempel, T.R., Mazo, I.B., von Andrian, U.H. Intravital Microscopy. Immunity. 21 (3), 315-329 (2004).

31. Lambert Emo, K. et al. Live Imaging of Influenza Infection of the Trachea Reveals Dynamic Regulation of CD8+ T Cell Motility by Antigen. PLOS Pathogens. 12 (9), e1005881 (2016).

32. Kjos, M. et al. Bright fluorescent Streptococcus pneumoniae for live-cell imaging of host-pathogen interactions. Journal of bacteriology. 197 (5), 807-818 (2015). 